\title{
TRASTORNO DEL ESPECTRO AUTISTA \\ EN ANOREXIA NERVIOSA: \\ INVISIBLES FRENTE AL ESPEJO
}

\section{AUTISTIC SPECTRUM DISORDER IN ANOREXIA NERVOSA: INVISIBLE ONES IN FRONT OF THE MIRROR}

\author{
Gema García Marco \\ Facultad de Psicología \\ Universidad Católica de Valencia San Vicente Mártir. Valencia. España \\ ORCID: https://orcid.org/0000-0002-1516-8450
}

Cómo referenciar este artículo/How to reference this article:

García Marco, G. (2020). Trastorno del Espectro Autista en Anorexia Nerviosa: Invisibles frente al espejo. Revista de Psicoterapia, 31(115), 63-76. https://doi.org/10.33898/rdp.v31i115.356

\begin{abstract}
Resumen
La relación entre el Trastorno del Espectro Autista(TE) y la Anorexia Nerviosa (AN) ha suscitado un creciente interés dadas las altas tasas de comorbilidad y la necesidad de adaptar los tratamientos existentes a esta población, ya que presentan peores resultados en los tratamientos estándar para el trastorno de conducta alimentaria. En este artículo se realiza una revisión descriptiva de diferentes aspectos relacionados con los TEA y la AN tratando de ofrecer una perspectiva sobre el tema que pueda ser de utilidad para la práctica clínica. En él se abordan aspectos epidemiológicos y perfiles neuropsicológicos, se profundiza en las particularidades del diagnóstico en estos casos y se exponen las propuestas de tratamiento.

Palabras clave: Trastorno Espectro Autista, Anorexia, Trastornos Alimentarios, Síndrome de Asperger, Terapia de Remediación Cognitiva.
\end{abstract}

\begin{abstract}
The relationship between Autism Spectrum Disorder (ASD) and Anorexia Nervosa (AN) has raised a growing interest given the high comorbidity rates and the need to adapt existing treatment to this population, since they present worse results in standard treatments for eating disorders. In this article, a descriptive review of different aspects related to ASD and $A N$ is carried out, trying to offer a perspective on the topic that may be useful for clinical practice. It covers epidemiological aspects and neuropsychological profiles, deepens the particularities of the diagnosis in these cases and presents treatment proposal.

Keywords: Autistic Spectrum Disorder, Anorexia, Eating Disorders, Asperger Syndrome, Cognitive Remediation Therapy.
\end{abstract}

Fecha de recepción: 9 de diciembre de 2019. Fecha de aceptación: 17 de enero de 2020. Correspondencia sobre este artículo:

E-mail: gema.garcia@ucv.es

Dirección postal: Avenida de la llustración, 4 (46100) Burjassot, Valencia. España

(C) 2020 Revista de Psicoterapia 


\section{Introducción}

La Anorexia Nerviosa (AN) se clasifica en el Manual Diagnóstico y Estadístico de los Trastorno Mentales (5. ${ }^{a}$ Ed.; DSM- V; American Psychiatric Association [APA], 2013) como uno de los trastornos alimentarios y de la ingestión de alimentos. Se caracteriza, entre otros síntomas, por un peso significativamente bajo, miedo a ganar peso o conducta persistente que interfiere en el aumento de peso, así como por una alteración en la forma en que uno mismo percibe su cuerpo, una influencia impropia del peso en la autoevaluación o falta de reconocimiento de la gravedad del bajo peso. La AN es un trastorno multideterminado, complejo y grave: posee la tasa más alta de mortalidad de todos los trastornos mentales (Harris y Barraclough, 1998).

Por otro lado, el Trastorno de Espectro Autista (TEA) es un trastorno del neurodesarrollo de inicio en la infancia, caracterizado por dificultades en la comunicación social y por patrones restrictivos y repetitivos de comportamiento. Puede darse con o sin déficit intelectual así como con o sin deterioro del lenguaje. En el $D S M-V$ (2013) desaparecen los subtipos que contemplaba el DSM-IV (1994) (el Síndrome de Asperger era por ejemplo, uno de ellos), ya que se reconoce un amplio abanico de fenotipos dentro del espectro. Por tanto, desaparece la nomenclatura de Trastorno de Asperger (dentro del espectro autista, este síndrome es uno de los que más se han estudiado en relación a la AN) pero eso no quiere decir que desaparezca el cuadro clínico en sí. Lo que cambia en el $D S M-V$ (2013) es la visión categorial, siendo considerado un trastorno dimensional, en función de las necesidades de apoyo requeridas. Las personas antes diagnosticadas como "Trastorno de Asperger" (Síndrome de Asperger, según la CIE-10, OMS, 1993) actualmente entrarían en la categoría diagnóstica de TEA de grado 1 especificando que no presentan déficit cognitivo ni deterioro del lenguaje.

Ya en los años ochenta Gillberg (1983) planteó la posibilidad de que pudiera haber un TEA subyacente en algunos casos de AN basándose en las semejanzas en sus perfiles cognitivos, sugiriendo incluso que compartían una vulnerabilidad genética que en interacción con factores ambientales podía manifestarse como AN en niñas y como TEA en niños durante la infancia. Desde entonces hasta ahora ha habido un creciente interés en la literatura científica por entender la relación existente entre estos dos trastornos dada la elevada presencia de TEA en AN y las dificultades subyacentes compartidas en el funcionamiento cognitivo, social y emocional (Westwood y Tchanturia, 2017). Las características que comparten los trastornos alimentarios (sobre todo la $\mathrm{AN}$ ) y el TEA incluyen los comportamientos repetitivos y limitados, la retirada social y las dificultades en entender los estados mentales ajenos (Nickel et al., 2019).

El TEA parece estar sobrerrepresentado en población con trastornos alimentarios. En su revisión sistemática, Westwood y Tchanturia (2017) encontraron que las personas con AN tenían más dificultades asociadas al TEA que los sujetos control. De otra revisión sistemática, se extraen datos de prevalencia de rasgos autistas en el 22,9\% de las pacientes diagnosticadas de AN (Huke, Turk, Saeidi, Kent y Morgan, 
2013). Es importante destacar que en la revisión anteriormente citada 6 de los 8 estudios considerados estaban basados en la misma muestra de población sueca, lo que podría haber sesgado los resultados. Resulta en cualquier caso complicado sacar conclusiones de prevalencia dados los cambios en los diagnósticos del DSM$I V$ (1994) al DSM-V (2013), los distintos instrumentos de medida utilizados y las dificultades en el diagnóstico de TEA en mujeres.

La mayoría de los estudios de comorbilidad se han realizado sobre todo en población adulta, existiendo muchos menos estudios en niños y adolescentes. En esta población, Kalyva (2009) concluye que aquellas chicas diagnosticadas de TEA en la adolescencia presentan un mayor riesgo de tener un trastorno alimentario que sus coetáneas, con datos que indican que un $27 \%$ de niñas con TEA presentaban síntomas dentro del espectro de los trastornos alimentarios

La comorbilidad entre TEA y AN está asociada a peor pronóstico (Nielsen, Anckarsater, Gillberg, Rastam y Wentz, 2015; Rhind et al, 2014) y a una peor respuesta al tratamiento ya que la rigidez y las dificultades de introspección características del TEA dificultan la respuesta a los programas terapéuticos tradicionales (Tchanturia et al., 2016)

\section{Alteraciones Alimentarias, TEA y Anorexia Nerviosa}

Las personas diagnosticadas de TEA suelen presentar con frecuencia alteraciones alimentarias. Partimos de la base de que estas alteraciones alimentarias no son necesariamente un trastorno alimentario, aunque si pueden suponer un factor de riesgo para padecerlo en el futuro (Rastam, 2008). El TEA puede cursar problemas alimentarios tales como; rechazo a alimentos, pica, rumiación, alimentación selectiva, neofobia (rechazo a alimentos nuevos), polidipsia y sobrealimentación, que tienden a persistir en la adolescencia y la edad adulta. De hecho, las alteraciones alimentarias formaron parte de las primeras descripciones de los indicadores tempranos del autismo (Ritvo y Freeman, 1978, citado por Rastam, 2008) así como el bajo peso: dos de los tres casos que originariamente describió Hans Asperger presentaban un peso por debajo de la media (Asperger, 1944, citado por Rastam, 2008).

La alimentación selectiva puede responder a diferentes factores, y en principio no tiene por qué afectar al peso, aunque en ocasiones sí a la vida social de la persona a quien le ocurre: unas veces se trata de la expresión de un comportamiento ritualizado mientras que otras responde a la híper sensibilidad de las personas del espectro autista a texturas, olores y sabores. Es posible que incluso el color pueda suponer un problema, y esto lleve a que la persona consuma alimentos monocromáticos.

Algunas de estas características son similares a las presentadas en los casos de Trastorno de Evitación/Restricción de alimentos, otro de los trastornos alimentarios categorizados en el $D S M-V$ (APA, 2013). Éste se caracteriza por falta de interés aparente por comer, evitación a causa de las características organolépticas del alimento o preocupación acerca de las consecuencias de comer que lleva a la persona al fracaso en el cumplimiento de sus necesidades nutritivas y/o energéticas. Para su 
diagnóstico se debe tener también en cuenta que el trastorno no se explique mejor por la falta de alimentos disponibles o por una práctica cultural asociada, que no se produzca en el curso de una anorexia o bulimia nerviosas y que no se atribuya a una afección médica u otro trastorno mental. El DSM-V (APA, 2013) especifica también que cuando este trastorno alimentario se da en el contexto de otra afección o trastorno, el diagnóstico de Trastorno de Evitación/Restricción de alimentos sólo debería realizarse si la gravedad excede a la que suele asociarse a la afección o trastorno y justifica la atención clínica adicional. Por tanto, si se da en alguien que ya tiene el diagnóstico de TEA, sólo debería diagnosticarse si se cumplen todos los criterios diagnósticos de ambos y si el trastorno alimentario requiere un tratamiento específico. Las personas con trastorno de evitación/restricción de alimentos difieren de las personas con AN en que las primeras no presentan alteraciones de imagen corporal ni miedo a ganar peso.

La prevalencia del trastorno de evitación/restricción de alimentos es significativamente mayor en niños TEA que en sus pares, lo que lleva a algunos autores a proponer que la presencia de alteraciones alimentarias graves en la infancia debería alertar de la posibilidad de que exista un TEA (Lucarelli, Pappas, Welchons y Augustyn, 2017).

Cuando existen sospechas de TEA y se observan alteraciones alimentarias del tipo que sea, el primer paso sería realizar un exhaustivo diagnóstico diferencial. Westwood y Tchanturia (2017) concluyen que podría ser beneficioso realizar de forma sistemática un cribado en búsqueda de un posible TEA en personas que se ponen en tratamiento por un trastorno alimentario, aunque posteriormente sea un clínico especializado en TEA quien realice el diagnóstico definitivo. Esta conclusión es compartida por otros autores como Nielsen et al. (2015) y Huke et al. (2013).

\section{Aspectos en Común TEA y AN}

El perfil neuropsicológico de los trastornos alimentarios ha sido objeto de estudio en las últimas décadas, siendo las características más consensuadas la debilidad en la coherencia central y la rigidez cognitiva. Más recientemente también se ha puesto el foco de la investigación en las dificultades socioemocionales, a través de estudios de la cognición social. Dentro del concepto de cognición social se encuentran varios subprocesos, incluido en ellos el de teoría de la mente. La AN y los TEA comparten algunas semejanzas conductuales, como por ejemplo rituales con la comida o dificultades para comer con otras personas en entornos sociales (Rastam 2008), comparten dificultades a nivel emocional como la alexitimia, es decir, la dificultad para identificar y expresar emociones (Mansour et al., 2016; Råstam, Gillberg, Gillberg y Johansson, 1997; Råstam, Gillberg, y Wentz, 2003) y podría ser que también compartan algunas características neurológicas tales como: débil coherencia central (dificultades para ver la globalidad en lugar de los detalles), problemas con la alternancia de tareas (la habilidad para cambiar de una tarea a otra de forma fluida, aspecto relacionado con la rigidez cognitiva) y dificultades 
con la teoría de la mente (la habilidad para atribuir estados mentales) (Nickel et al., 2019). De forma específica:

- El concepto de dificultad con la alternancia de tareas hace referencia a los problemas para utilizar un estilo de pensamiento flexible que posibilite alternar entre operaciones, acciones, tareas...etc. Las dificultades en esta área llevan a la rigidez cognitiva, que puede manifestarse a través de la tendencia a poner en marcha estrategias inflexibles de solución de problemas que complica la adaptación a las circunstancias vitales, cambiantes por naturaleza. La rigidez cognitiva también está relacionada con el tipo de pensamiento "todo-nada" y las tendencias perfeccionistas (Treasure y Schmidt, 2013). Escobar-Koch (2012) recoge diferentes investigaciones que demuestran flexibilidad cognitiva reducida en pacientes con anorexia. Estas dificultades no mejoran con la ganancia de peso, se han encontrado en familiares de personas con AN y se mantienen en pacientes recuperadas (Tchanturia et al., 2004), lo que sugiere que es un rasgo estable. Esta dificultad se encuentra también en personas con TEA.

- Cuando hablamos de coherencia central nos referimos a la tendencia a procesar la información focalizando en los detalles en lugar de tener una visión amplia de conjunto. Existe evidencia de que las personas con AN prestan excesiva atención a los detalles y son menos eficientes en el procesamiento de información de forma global. Este tipo de procesamiento podría explicar los intereses restringidos y cómo las pacientes perciben su cuerpo: de forma hiperfocalizada en aquellas partes que no les gustan de sí mismas y con una gran dificultad para valorarlo de forma global. Sabemos que los problemas de coherencia central son más pronunciados durante la fase aguda de la enfermedad (Nickel et al., 2019) y parece ser un rasgo estable ya que está presente en pacientes recuperadas (Lang, López, Sthal, Tchanturia y Treasure, 2014). Estos problemas de coherencia central también se encuentran en personas con TEA.

- Para finalizar, la teoría de la mente(Theory of Mind,ToM) hace referencia a las habilidades para percibir los estados afectivos de los demás y de uno mismo, así como de interpretar y hacer inferencias en situaciones sociales que ayuden a predecir el comportamiento de los demás. En personas con AN existen dificultades en las tareas relacionadas con la ToM, así como en personas TEA. En la AN se está estudiando la importancia del índice de masa corporal (IMC) en el desempeño en las tareas que evalúan este constructo para averiguar el papel que puede tener el estado de privación de alimentos ya que parece que esta dificultad podría estar limitada a la fase aguda de la enfermedad como consecuencia de la inanición (Nickel et al., 2019). Los resultados no son concluyentes todavía, como tampoco parecen serlo en mujeres TEA ya que la mayoría de los estudios que encuentran dificultades de personas del espectro autista en la teoría de la 
mente se han realizado con varones, por lo que se sabe muy poco acerca de la ToM en niñas y mujeres TEA (Sedgewick et al., 2019).

En una revisión reciente de la literatura (Westwood, y Tchanturia, 2017) se concluye que no está claro todavía si la presencia de síntomas TEA en AN responde a un trastorno del neurodesarrollo subyacente, si son agravados por el propio trastorno alimentario o si son meras semejanzas en el funcionamiento emocional y socio-cognitivo. En la misma línea, en otra revisión de 2019 (Nickel et al., 2019) se concluye que la falta de estudios longitudinales no permite aclarar si los déficits neurocognitivos son un factor de riesgo o una consecuencia del TCA.

Desde una perspectiva cualitativa, Kinnaird, Norton, Stewart y Tchanturia (2018) recogen experiencias en primera persona de quienes tienen el doble diagnóstico: llevaron a cabo entrevistas semiestructuradas con mujeres con AN y el diagnóstico de TEA o elevados rasgos autistas en el AQ-10 (Short Autism Specrum Quotient, Allison, Auyeung y Baron-Cohen, 2012) o el ADOS (Autism Diagnosis Observation Schedule, Lord et al., 2000). Las participantes en el estudio sentían que su condición autista y su AN estaban profundamente relacionadas: una de las formas en las que ellas percibían esta relación es a través de la rigidez y la inflexibilidad que una vez volcadas en la comida, apenas permitían cambios en las rutinas establecidas. Otro dato interesante es que estas mujeres describieron que el deseo de perder peso y los problemas de imagen corporal no fueron determinantes para el desarrollo de su AN, sus motivaciones iban más en la línea de la necesidad de control, la rigidez en sus patrones, dificultades sensoriales, confusión social, la lucha que supone para ellas relacionarse con los demás, las dificultades en organizar temas relacionados con la compra o la cocina, el ejercicio como forma de estimulación y el trastorno alimentario como un foco de interés. Para muchas de las participantes del estudio, la AN se convirtió en la forma de afrontar sus dificultades (Kinnaird et al., 2018).

\section{TEA y AN, ¿una cuestión de género?}

El TEA se diagnostica hasta tres o cuatro veces más en el sexo masculino que en el femenino, con ratios del 3/1, según algunos autores (Loomes, Hull y Mandy, 2017) y del $4 / 1$ según otros (Cheslack-Postava y Jordan-Young, 2012). Estos índices son incluso mayores en el caso del Síndrome de Asperger (Gillberg, Cederlund, Lamberg y Zeijlon, 2006). Por todo ello, la investigación hasta el momento se ha realizado principalmente con varones, lo que ha supuesto que se produzca un "sesgo de género" tanto en las descripciones clínicas como en los criterios diagnósticos ya que a día de hoy sabemos que éstos son diferentes en hombres y mujeres (Van Wijngaarden-Cremers, van Eeten, Groen, Van Deurzen, Oosterling y Van der Gaag, 2014). Esta realidad ha significado que en muchos casos se produzca un retraso y/o un infradiagnóstico de TEA en mujeres, que junto con las presiones socioculturales, las hace vulnerables a desarrollar trastornos mentales secundarios, incluyendo laAN (Westwood et al., 2016; Westwood y Tchanturia, 2017) y ha supuesto también que con frecuencia a lo largo de los años vayan recibiendo otras etiquetas diagnósticas 
variadas, tales como fobia social o trastorno límite de personalidad (Attwood, 2007, citado en Van Wijngaarden-Cremers et al., 2014; para una revisión exhaustiva ver Montagut, Romero y Andrés, 2018).

Las mujeres TEA suelen mostrar mayor motivación social (Head et al., 2014), menos estereotipias y menos intereses restrictivos que los hombres (Van Wijngaarden-Cremers et al., 2014; Frazier et al., 2014) y con frecuencia sus intereses están más alineados con los estereotipos de género por lo que son más difícilmente detectables (Hiller et al., 2015). Los estudios sugieren que las mujeres TEA analizan los comportamientos sociales de los demás y encuentran formas de enmascarar su autismo. Estas formas de "camuflaje" pueden funcionar como factor protector a nivel social, pero contribuyen al infradiagnóstico si estas mujeres se encuentran con profesionales que consideran que no cuadran en el perfil esperado, es decir, pasan "por debajo del radar" (Lawson 2017; Zener, 2019). Estos intentos de camuflar su condición pueden traer también otras dificultades como problemas de identidad ("no sé quién soy realmente"), elevados niveles de ansiedad y una enorme fatiga, que hace que en muchas ocasiones prefieran o necesiten estar a solas para liberar la sobrecarga (Westwood y Tchanturia, 2017; Westwood, Mandy, Simic y Tchanturia, 2018).

Esta situación en la que se encuentran las mujeres del espectro autista es grave y tiene consecuencias: las mujeres diagnosticadas de TEA en comparación con los hombres, presentan tasas de depresión significativamente más altas, así como riesgo suicida (Cassidy et al., 2014).Un diagnóstico a tiempo funcionaría como un factor protector, aliviando su lucha interna, y permitiendo que un proceso terapéutico las acompañe a conocerse mejor a sí mismas, así como a encontrar las maneras de adaptarse a un mundo normotípico, que les resulta complicado y confuso, y del que no se sienten parte. Es común escuchar de ellas afirmaciones como "me he sentido diferente toda mi vida": esta conciencia de la diferencia y la realidad de que habitualmente son personas que se han sentido muy solas, sobre todo en la adolescencia, hacen que estas mujeres crezcan con sentimientos de inadecuación, problemas de autoestima, sensación de inseguridad y una profunda vergüenza ("tengo que fingir ser otra persona para ser aceptada, por lo que debe haber algo malo en mi"), que las llevan a sentir constantemente la sensación de que "no son suficientes" y que "nada de lo que hacen es nunca suficiente". En ocasiones los síntomas de AN pueden ser para las personas TEA un intento de solución a su angustiosa situación. En palabras de una paciente con doble diagnóstico (reproducido con su autorización): "Siempre me he sentido diferente, con unas necesidades fuera de la normalidad y esto era algo que no podía controlar. Esa inseguridad me llevó a una búsqueda de control de mi cuerpo que se me escapó de las manos”.

Las chicas con autismo tienen un alto deseo de complacer y de encajar, por lo que son hipersensibles a las demandas del entorno y fácilmente manipulables, con elevados deseos de cumplir las normas sociales (Lawson, 2017; Yaull-Smith, 2008). En este contexto no es infrecuente que algunas pacientes con el doble diag- 
nóstico nos cuenten en consulta que éste fue uno de los detonantes de su trastorno alimentario: el deseo de encajar en una sociedad que ensalza la delgadez guiadas por el pensamiento mágico que dice que una vez sean delgadas, serán aceptadas y todos sus problemas se solucionarán.

Sabemos también que las mujeres TEA tienen un alto riesgo de vivir alguna situación traumática a lo largo de su vida; las experiencias de bullying son muy comunes, y pueden llevar a la persona a sufrir elevados niveles de ansiedad, depresión, a recurrir a autolesiones y a formarse un autoconcepto negativo (Cappadocia, Weiss y Pepler, 2012). Por si esto fuera poco, las niñas con TEA tienen tres veces más probabilidades de sufrir un abuso sexual que las niñas normotípicas de su edad (Ohlsson, Lichtenstein, Långström y Pettersson, 2018). Tanto las experiencias de acoso escolar como los abusos sexuales pueden ser factores predisponentes y/o precipitantes para desarrollar un trastorno alimentario en el futuro (Lie, Ro y Bang, 2019; Madowitz, Matheson y Liang, 2015).

Por todo lo comentado anteriormente, en ocasiones nos encontraremos un TEA no diagnosticado en personas que lleguen a consulta pidiendo ayuda por un trastorno alimentario. Es importante que sepamos reconocer este tipo de perfil para poder realizar un correcto diagnóstico y ajustar la intervención a sus necesidades. (Para una revisión exhaustiva al respecto de las dificultades para acceder al diagnóstico de mujeres TEA el lector puede revisar el artículo de Zener, 2019).

\section{La Complejidad del Diagnóstico}

El diagnóstico de TEA es fundamental para poder ofrecer un tratamiento ajustado a las particularidades del caso, pero es sin duda un reto complicado por varios motivos.

Como se comentaba anteriormente, el TEA está generalmente infradiagnosticado en mujeres. Esto se debe a varios motivos: por un lado, los instrumentos de evaluación existentes y los criterios diagnósticos del DSM-V no contemplan las diferencias de género (diferencias que son tan claras que se ha llegado a proponer que pueda haber un fenotipo autista diferente en mujeres) y por otro, las mujeres con TEA tienden a pasar desapercibidas, ya que presentan menos comportamientos estereotipados y restrictivos que los hombres y generan "estrategias de camuflaje".

Pero a su vez, los síntomas de AN pueden enmascarar el cuadro de TEA cuando se manifiestan con extrema rigidez y obsesiones focalizadas en el cálculo de calorías, el control de peso o el ejercicio (Mandy y Tchanturia 2015; Westwood y Tchanturia, 2017). Establecer claramente cuál es la relación se complica aún más si tenemos en cuenta que algunas personas sin TEA pueden mostrar comportamientos asociados a éste durante las fases agudas de la anorexia debido a las consecuencias psicológicas del bajo peso tales como: irritabilidad, estado de ánimo deprimido, menor iniciativa e introversión, menos flexibilidad cognitiva, menor motivación social así como una mayor obsesividad (Keys, 1950, citado en Huke et al., 2013; Nickel et al, 2019; Westwood et al., 2018). Es por tanto fundamental 
distinguir si los síntomas alimentarios que presenta alguien que llega a consulta son parte del diagnóstico subyacente de TEA (los problemas con la comida son una manifestación más de los síntomas nucleares del TEA) o si por el contrario existe una comorbilidad clara, ya que cumplen criterios diagnósticos de ambos trastornos. Algo que nos puede ayudar es tener siempre presente que para realizar el diagnóstico de TEA los síntomas deben estar presentes desde la infancia, antes de que aparezca el trastorno alimentario, y además no mejorar significativamente tras la recuperación ponderal. Es importante realizar una historia del desarrollo, y trabajar en equipo: lo ideal sería que un especialista en trastornos alimentarios y un especialista en TEA realizaran de forma conjunta el diagnóstico y pudieran trabajar en equipo durante el tratamiento (Dudova et al., 2015).

\section{Implicaciones para el Tratamiento}

A lo largo de las diferentes investigaciones llevadas a cabo, se ha comprobado que las características patológicas nucleares de los TEA pueden estar implicadas no sólo en el inicio sino también en el mantenimiento de los TCA (Mandy y Tchanturia, 2015). La comorbilidad entre TEA y AN está asociada a peor pronóstico (Rhind et al., 2014), a una peor respuesta al tratamiento, así como a la necesidad de tratamientos más intensivos (Westwood y Tchanturia, 2017) ya que la rigidez y las dificultades de introspección y comunicación características del TEA dificultan la respuesta a los programas terapéuticos tradicionales para trastornos alimentarios (Dudova et al., 2015; Tchanturia et al., 2016). La presencia de rasgos TEA agudos en personas con AN están asociados a sintomatología más grave, peor pronóstico y peores resultados en el tratamiento (Nielsen et al., 2015; Rhind et al, 2014; Tchanturia, Adamson, Leppanen y Westwood, 2017; Tchanturia, Larsson y Adamson, 2016). En este momento existe poca literatura que se centre en las necesidades de esta población, por lo que escuchar a quienes les ponen palabras en primera persona es de gran ayuda para los clínicos: en el estudio de Kinnaird (2018) citado anteriormente, las mujeres diagnosticadas con AN y TEA que habían accedido a tratamientos específicos para su trastorno alimentario relataban haberse sentido vistas como pacientes difíciles, perezosas, cabezotas o poco cooperativas por sus terapeutas dado su TEA no diagnosticado en ese momento. Este hecho llevaba a que se empobreciera la relación terapéutica y con el tiempo les había llevado en algunos casos a abandonar el tratamiento. Estas pacientes refirieron también dificultades para adaptarse a entornos terapéuticos de hospitalización dadas sus dificultades sociales. En esta misma línea, las personas con problemas sensoriales con su alimentación describieron haber tenido grandes problemas con los programas de renutrición que no tenía en cuenta su condición autista ni por tanto sus particularidades sensoriales. Sus dificultades principales incluían: sensibilidad aumentada al sabor, olfato y textura, aversión a mezclar determinados alimentos y la necesidad de que la comida estuviera a una temperatura determinada. Las participantes describían una enorme frustración cuando conductas relacionadas con estos aspectos se etiquetaban como 
síntoma del trastorno alimentario. Para finalizar, estas mujeres relataban su necesidad de tratamientos más largos, dado el reto que supone para ellas salir de la rigidez y las rutinas asociadas a su condición autista.

Las dificultades subyacentes relacionadas con la coherencia central pueden ampliar los rasgos perfeccionistas y obsesivo-compulsivos de las personas con AN dificultando su adherencia a tratamientos en los que se enfatiza el cambio a nivel conductual, por lo que los tratamientos podrían necesitar centrarse primero en las características cognitivas propias de este tipo de pacientes (Tchanturia et al. 2013). Para dar respuesta a estas dificultades, surge el interés en la terapia de remediación cognitiva como forma de abordaje posible en estos casos.

La terapia de remediación cognitiva (TRC), que fue inicialmente desarrollada para personas con lesión cerebral o daño cerebral adquirido, aborda características que comparten ambos diagnósticos (rigidez cognitiva y problemas de coherencia central) y se ha demostrado eficaz en casos de AN (Davies et al., 2012; Tchaturia, Davies y Campbell, 2007; Tchanturia et al., 2013). En ella se trabajan los procesos cognitivos (cómo piensan la persona) más que el contenido de los pensamientos (qué piensa la persona) (Escobar-Koch, 2013). El objetivo de la TRC es que las pacientes reflexionen a través del descubrimiento guiado sobre sus propios estilos de pensamiento y cómo éstos les afectan en su vida cotidiana. Se trata de que reconozcan sus estilos de pensamiento inflexibles para después trabajar sobre las áreas de su vida en que presentan comportamientos rígidos (comida, cuerpo, ejercicio... etc.), así como que reconozcan su excesiva atención a los detalles y promover el desarrollo del pensamiento más global en su vida cotidiana (por ejemplo: pasar de enfocarse en las calorías de los alimentos a tener una visión más amplia del alimento). Esta forma de abordaje puede ser menos amenazante en fases iniciales del tratamiento, ya que no se tratan directamente aspectos relacionados con la comida, el cuerpo o el peso. Los elementos principales de las sesiones de TRC son las siguientes: realización de actividades en las que emergen los estilos cognitivos predominantes de la paciente, identificación de fortalezas y debilidades de las estrategias utilizadas y cómo se aplican a su vida cotidiana, aprendizaje de estrategias cognitivas nuevas y discusión acerca de posibles tareas o experimentos conductuales entre sesiones para practicar las nuevas estrategias cognitivas aprendidas (Escobar-Koch, 2013). Las tareas que se utilizan para trabajar sobre la rigidez cognitiva y que buscan aumentar la flexibilidad en el estilo de pensamiento son, entre otras, tareas de tipo "Stroop" en sus distintas versiones y tareas de atención alternante. Los ejercicios de la TRC para trabajar el pensamiento global están centrados, sobre todo, en ejercicios de figuras complejas o actividades relacionadas con extraer la idea principal de un texto (Escobar-Koch, 2013). LA TRC es una intervención breve que puede funcionar como trabajo introductorio previo a otras formas de psicoterapia (Tchanturia, Lloyd y Lang, 2013). Tchanturia, Davies, Reeder y Wykes (2010) realizan una exhaustiva descripción de la intervención desde la TRC, incluyendo ejercicios listos para la aplicación individual y grupal así como descripción de casos, muy 
útil para la práctica clínica.

Por último, una reflexión respecto al significado de recuperación en personas TEA: algunas formas de funcionamiento en personas del espectro autista tales como la rigidez o la sensibilidad también referida a lo alimentario puede que persistan tras la recuperación. Por tanto, los terapeutas que tratan con personas TEA deben tener en cuenta lo que sus pacientes consideran que es la recuperación para ellos; identificar qué conductas responden al trastorno alimentario y pueden ser abordadas en el tratamiento, y cuáles tienen sus raíces en la condición autista y requerirían más bien un proceso de adaptación y aceptación (Kinnaird et al., 2017).

\section{Conclusiones}

Está ampliamente demostrada la estrecha relación entre el TEA y la AN, aunque sobre la mesa quedan aún más preguntas que respuestas. Dada la elevada comorbilidad entre ambos y la notable prevalencia de alteraciones alimentarias en personas TEA, sería de gran ayuda usar herramientas de cribado con cualquier persona que llegue a consulta para ponerse en tratamiento por su trastorno alimentario, para confirmar o descartar la presencia de un posible TEA y remitir, si es necesario, a una evaluación exhaustiva por un profesional especializado. Es por tanto fundamental que los clínicos podamos ver más allá de los síntomas, es un reto conseguir que las mujeres TEA se sientan por fin vistas: se estima que el 70\% de personas TEA podrían tener un trastorno mental comórbido (Montagut et al., 2918) y la realidad actual es que es frecuente que pasen por diferentes consultas de salud mental recibiendo diagnósticos sólo de sus patologías asociadas, permaneciendo invisible el TEA. Diagnosticar a tiempo es no sólo prevención en el caso de niñas y adolescentes TEA, sino también la clave para ajustar los tratamientos a las necesidades de esta población. La detección temprana y la adaptación de las intervenciones también supone compartir un modelo mental que permita a los equipos terapéuticos establecer una relación terapéutica "bien tratante" en la que las personas TEA se sientan reconocidas, entendidas y validadas.

\section{Referencias Bibliográficas}

Allison, C., Auyeung, B. y Baron-Cohen, S. (2012). Towards brief 'red flags' for autism screening: the short Autism Spectrum Quotient and the short Quantitative Checklist for Autism in toddlers in 1000 cases and 3000 controls. Journal of the American Academy of Child and Adolescent Psychiatry, 51(2), $202-212$. https://doi.org/10.1016/j.jaac.2011.11.003

American Psychiatric Association (2013). The diagnostic and statistical manual of mental disorders, (5 $5^{\text {th }}$ ed.). Arlington, VA: American Psychiatric Association.

American Psychiatric Association (1994). Manual diagnóstico y estadístico de los trastornos mentales, (4 ${ }^{\text {th }}$ ed.). Barcelona, España: Masson.

Attwood, T. (2007). The complete guide to Asperger's syndrome. Londres, Reino Unido: Jessica Kingsley.

Cappadocia, M. C., Weiss, J. A. y Pepler, D. (2012). Bullying experiences among children and youth with autism spectrum disorders. Journal of Autism and Developmental Disorders, 42(2), 266-77. https://doi. org/10.1007/s10803-011-1241-x 
Cassidy, S., Bradley, P., Robinson, J., Allison, C., McHugh, M. y Baron-Cohen, S. (2014). Suicidal ideation and suicide plans or attempts in adults with Asperger's Syndrome attending a specialist diagnostic clinic: a clinical cohort study. Lancet Psychiatry, 1(2), 142-147. https://doi.org/10.1016/S2215-0366(14)70248-2

Cheslack-Postava, K. y Jordan-Young, R. M. (2012). Autism spectrum disorders: toward a gendered embodiment model. Social science \& medicine, 74(11), 1667-1674. https://doi.org/10.1016/j.socscimed.2011.06.013

Davies, H., Fox, J., Naumann, U., Treasure, J., Schmidt, U. y Tchanturia, K. (2012). Cognitive remediation and emotion skills training in anorexia: an observational study using neuropsychoogical outcomes. European eating disorders review, 20(3), 211-217. https://doi.org/10.1002/erv.2170

Dudova, I., Kocourkova, J. y Koutec, J. (2015). Early-onset anorexia nervosa in girls with Asperger syndrome. Neuropsychiatric Disease and Treatment, 11, 1639-1643. https://dx.doi.org/10.2147\%2FNDT.S83831

Escobar-Koch, T. (2013). Terapia de remediación cognitiva para anorexia nerviosa. Revista Chilena Psiquiatría y Neurología de la Infancia y la Adolescencia, 23(3), 177-189.

Frazier, T. W., Georgiades, S., Bishop, S. L. y Hardan, A. Y. (2014). Behavioral and cognitive characteristics of females and males with autism in the Simons simplex collection. Journal of the American Academy of Child and Adolescent Psychiatry, 53(3), 329-340. https://doi.org/10.1016/j.jaac.2013.12.004

Gillberg, C. (1983). Are autism and Anorexia Nervosa related? British Journal of Psychiatry, 142(4), 428. https:// doi.org/10.1192/bjp.142.4.428b

Gillberg, C., Cederlund, M., Lamberg, K. y Zeijlon, L. (2006). Brief report: “the autism epidemic”. The registered prevalence of autism in a Swedish urban area. Journal of autism and developmental disorders, 36, 429. https://doi.org/10.1007/s10803-006-0081-6

Harris, E. C. y Barraclough, B. (1988). Excess mortality of mental disorder. British Journal of Psychiatry, 173(1), 11-53. https://doi.org/10.1192/bjp.173.1.11

Hiller, R. M., Young, R. L. y Weber, N. (2015). Sex differences in pre-diagnosis concerns for children later diagnosed with autism spectrum disorder. Autism, 20(1), 75-84. https://doi.org/10.1177/1362361314568899

Head, A. M., McGillivray, J. A. y Stokes, M. A. (2014). Gender differences in emotionality and sociability in children with autism spectrum disorders. Molecular Autism, 5(1), 19. https://doi.org/10.1186/2040- 2392-5-19

Huke, V., Turk, J., Saeidi, S., Kent, A. y Morgan, J. F. (2013). Autism spectrum disorders in eating disorder populations: a systematic review. European Eating Disorders Review, 21(5), 345-351. https://doi.org/10.1002/erv.2244

Kalvya, E. (2009). Comparison of eating attitudes between adolescent girls with and without asperger syndrome. Daughters and mothers' report. Journal of Autism and Developmental Disorders, 39(3), 480-486. https:// doi.org/10.1007/s10803-008-0648-5

Kinnaird, E., Norton, C., Stewart, C. y Tchanturia, K. (2018). Same behaviours, different reasons: what do patients with co-occurring anorexia and autism want for treatment? International Review of Psychiatry, 31(4), 308-317. https://doi.org/10.1080/09540261.2018.1531831

Lang, K., Lopez, C., Stahl, D., Tchanturia, K. y Treasure, J. (2014). Central coherence in eating disorders: An updated systematic review and meta-analysis. The World Journal of Biological Psychiatry, 15(8), 586-598. https://doi.org/10.3109/15622975.2014.909606

Lawson, W. (2017). Women \& girls on the autism spectrum: A Profile. Journal of Intellectual Disability-Diagnosis and Treatment, 5(3), 90-95. http://dx.doi.org/10.6000/2292-2598.2017.05.03.4

Lie, S.O., Ro, O. y Bang, L. (2019). Is bullying and teasing associated with eating disorders? A systematic review and meta-analysis. International journal of eating disorders, 52(5), 1-18. https://doi.org/10.1002/eat.23035

Loomes, R., Hull, L. y Mandy, W. P. L. (2017). What is the male-to-female ratio in autism spectrum disorder? A systematic review and meta-analysis. Journal of the American Academy of Child \& Adolescent Psychiatry, 56(6), 466-474. https://doi.org/10.1016/j.jaac.2017.03.013

Lord., C., Risi, S., Lambrecht, L., Cook, E. H., Leventhal, B., DiLavore, P. C., ... Rutter, M. (2000). The Autism Diagnostic Observations Schedule-Generic: A Standar Measure of Social an Communication Deficits Associated with the Spectrum of Austism. Journal of Autims and Developmental Disorders, 30, 205-223. https://doi.org/10.1023/A:1005592401947

Lucarelli, J., Pappas, D., Welchons, L. y Augustyn, M. (2017). Autism spectrum disorder and avoidant/restrictive food intake disorder. Journal of Developmental y Behavioral Pediatrics, 38(1), 79-80. https://doi.org/ 10.1097/DBP.0000000000000362

Madowitz, J., Matheson, B. y Liang, J. (2015). The relationship between eating disorders and sexual trauma. Eating and weight disorders-Studies on anorexia, bulimia and obesity, 20(3), 281-293. https://doi.org/10.1007/ s40519-015-0195-y

Mandy, W. y Tchanturia, K. (2015). Do women with eating disorders who have social and flexibility difficulties really have autism? A case series. Molecular Autism, 6(1), 6. https://doi.org/10.1186/2040-2392-6-6 
Mansour, S., Rozenblat, V., Fuller Tyszkiewicz, M., Paganini, C., Treasure, J. y Krug, I. (2016). Emotions mediate the relationship between autistic traits and disordered eating: A new autistic emotional model for eating pathology. Psychiatry Research, 245, 119- 126. https://doi.org/10.1016/j.psychres.2016.08.021

Montagut, M., Romero, R. y Andrés, M. (2018). Influencia del sesgo de género en el diagnóstico de trastorno de espectro autista: una revisión. Escritos de Psicología, 11(1), 42-54. http://dx.doi.org/10.5231/psy. writ.2018.2804

Nickel, K., Maier, S., Endres, D., Joos, A., Maier, V., Tebarz, L. y Zeeck, A. (2019) Systematic Review: Overlap between eating, autism spectrum and attention-deficit/hyperactivity disorder. Frontiers in psychiatry, 10(708). https://doi.org/10.3389/fpsyt.2019.00708

Nielsen, S., Anckarsater, H., Gillberg, C., Rastam, M. y Wentz, E. (2015). Effects of autism spectrum disorders on outcome in teenage-onset anorexia nervosa evaluated by the Morgan-Russell outcome assessment schedule: Acontrolled community-based study. Molecular Autism, 6, 14.https://doi.org/10.1186/s13229-015-0013-4

Ohlsson, G., Lichtenstein, P., Långström, N. y Pettersson, E. (2018). Childhood neurodevelopmental disorders and risk of coercive sexual victimization in childhood and adolescence - a population-based prospective twin study. Journal of Child Psychology and Psychiatry, and Allied Disciplines, 59(9), 957-965. https:// doi.org/10.1111/jepp. 12884

Organización Mundial de la Salud (1993). Clasificación Internacional de las Enfermedades (CIE-10). Ginebra, Suiza: OMS.

Råstam, M., Gillberg, C. y Wentz, E. (2003). Outcome of teenage-onset anorexia nervosa in a Swedish-community based sample. European Child \& Adolescent Psychiatry, 12(1), 78-90. https://doi.org/10.1007/s00787003-1111-y

Rastam, M. (2008). Eating disturbances in autism spectrum disorders with focus on adolescent and adult years. Clinical Neuropsychiatry, 5(1), 31-42.

Rhind, Ch., Bonfioli, E., Hibbs, R., Goddard, E., Macdonald, P., Gowers, S., ... Treasure, J. (2014). An examination of autism spectrum traits in adolescents with anorexia nervosa and their parents. Molecular Autism, 5, 56-65. https://doi.org/10.1186/2040-2392-5-56

Sedgewick, F., Leppanen, J., Goh, F., Hayward, H., Happé, F. y Tchanturia, K. (2019). Similarities and Differences in Theory of Mind Responses of Patients With Anorexia Nervosa With and Without Autistic Features. Frontiers in Psyquiatry, 10, 318. https://doi.org/10.3389/fpsyt.2019.00318

Stewart, C. S., McEwen, F. S., Konstantellou A, Eisler, I. y Simic, M. (2017). Impact of ASD Traits on treatment outcomes of eating disorders in girls. European Eating Disorders Review, 25(2), 123-128. https://doi. org/10.1002/erv.2497

Tchanturia, K., Anderluh, M. B., Morris, R. G., Rabe-Hesketh, S., Collier, D. A., Sanchez, P., ... Treasure, J. L. (2004). Cognitive flexibility in anorexia nervosa and bulimia nervosa. Journal of International Neuropsychological Society, 10(4), 513-520. https://doi.org/10.1017/S1355617704104086

Tchanturia, K., Davies, H. y Campbell, I. C. (2007). Cognitive remediation therapy for patients with anorexia nervosa: Preliminary findings. Annals of General Psychiatry, 6, 14. https://doi.org/10.1186/1744-859X-6-14

Tchanturia, K., Davies, H., Reeder, C. y Wykes, T. (2010). Cognitive Remediation Therapy for Anorexia Nervosa. Recuperado de: http:/www.national.slam.nhs.uk/wp-content/uploads/2014/04/Cognitive-remediationtherapy-for-Anorexia-Nervosa-Kate-Tchantura.pdf

Tchanturia, K., Larsson, E. y Adamson, J. (2016). How anorexia nervosa patients with high and low autistic traits respond to group Cognitive Remediation Therapy. BMC Psychiatry, 16, 334. https://doi.org/10.1186/ s12888-016-1044-x

Tchanturia, K., Lloyd, S. y Lang, K. (2013). Cognitive remediation therapy for anorexia nervosa: Current evidence and future research directions. International Journal of Eating Disorders, 46(5), 492-495. https:// doi.org/10.1002/eat.22106.

Tchanturia, K., Lounes, N. y Holttum, S. (2014). Cognitive remediation in anorexia nervosa and related conditions: a systematic review. European Eating Disorders Review, 22(6), 454-462. https://doi.org/10.1002/erv.2326

Tchanturia, K., Adamson, J., Leppanen, J. y Westwood, H. (2017). Characteristics of autism spectrum disorder in anorexia nervosa: A naturalistic study in an inpatient treatment programme. Autism, 23(1), 123-130. https://doi.org/10.1177\%2F1362361317722431

Treasure, J. y Schmidt, U. (2013). The cognitive-interpersonal maintenance model of anorexia nervosa revisited: A summary of the evidence for cognitive, socio-emotional and interpersonal predisposing and perpetuating factors. Journal of Eating Disorders, 1, 13. https://doi.org/10.1186/2050-2974-1-13 
Van Wijngaarden-Cremers, P. J. M., van Eeten, E., Groen, W. B., Van Deurzen, P. A., Oosterling, I. J. y Van der Gaag, R. J. (2014). Gender and Age Differences in the Core Triad of Impairments in Autism Spectrum Disorders: A Systematic Review and Meta-analysis. Journal Autism Developmental Disorders, 44, 627-635. https://doi.org/10.1007/s10803-013-1913-9

Westwood, H., Eisler, I., Mandy, W., Leppanen, J., Treasure, J. y Tchanturia, K. (2016). Using the Autism-Spectrum Quotient to Measure Autistic Traits in Anorexia Nervosa: A Systematic Review and Meta-Analysis. Journal Autism Developmental Disorders, 46, 964-977. https://doi.org/10.1007/s10803-015-2641-0

Westwood, H. y Tchanturia, K. (2017). Autism Spectrum Disorder in Anorexia Nervosa: An Updated Literature Review. Curr Psychiatry Rep, 19, 41. https://doi.org/10.1007/s11920-017-0791-9

Westwood, H., Mandy, W. y Tchanturia, K. (2017). Clinical evaluation of autistic symptoms in women with anorexia nervosa. Molecular Autism, 8, 12. https://doi.org/10.1186/s13229-017-0128-x

Westwood, H., Mandy, W., Simic, M. y Tchanturia, K. (2018). Assessing ASD in Adolescent Females with Anorexia Nervosa using Clinical and Developmental Measures:a Preliminary Investigation. Journal of Abnormal Child Psychology, 46, 183-192. https://doi.org/10.1007/s10802-017-0301-x

Yaull-Smith, D. (2008, marzo). Girls on the spectrum. Communication. National Autistic Society. Recuperado de: https://www.autismhampshire.org.uk/assets/uploads/Dale-Yaull-Smith-Communication-Spring-2008.pdf

Zener, D. (2019). Journey to diagnosis for women with autism. Advances in autism, 5(1), 2-13. https://doi. org/10.1108/AIA-10-2018-0041 